\title{
Resultados econômicos da atividade leiteira na região de Lavras (MG) nos anos 2004 e 2005: um estudo multicasos
}

\author{
[Economical results of milk production systems in Lavras region (MG) throughout the years \\ 2004 and 2005: a multicase study]

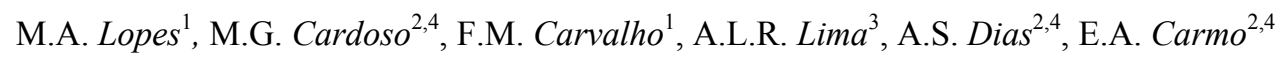 \\ ${ }^{1}$ Universidade Federal de Lavras \\ Caixa postal 3037 \\ 37200-000 - Lavras, MG \\ ${ }^{2}$ Aluno de graduação - UFLA - Lavras, MG \\ ${ }^{3} \mathrm{PUC}$ - Minas - Arcos, MG \\ ${ }^{4}$ Bolsista da FAPEMIG
}

\section{RESUMO}

Estudou-se a rentabilidade da atividade leiteira de 17 propriedades da região de Lavras/MG e identificaram-se os componentes que exerceram maior influência sobre os custos finais da atividade. Os dados foram coletados mensalmente durante o período de janeiro de 2004 a dezembro de 2005. Considerou-se a margem bruta, a margem líquida e o resultado (lucro ou prejuízo) como indicadores de eficiência econômica. Os itens componentes do custo operacional efetivo que exerceram maior influência sobre os custos da atividade leiteira foram, em ordem decrescente, alimentação (55,9\%), mão-de-obra $(19,1 \%)$, despesas diversas $(11,3 \%)$, energia $(7,1 \%)$, sanidade $(4,9 \%)$, ordenha $(0,74 \%)$, impostos $(0,74 \%)$ e inseminação artificial $(0,23 \%)$. A margem líquida e o resultado positivo indicam que os pecuaristas têm condições de produzir a médio e a longo prazo, com conseqüente capitalização. Os resultados do estudo não podem ser extrapolados por não se tratar de uma amostra probabilística.

Palavras-chave: bovinocultura de leite, análise de rentabilidade, custo de produção, ponto de equilíbrio

\begin{abstract}
It was studied the profitability of the milk production in 17 properties in the region of Lavras (MG), and the components that had the greatest influence on the final costs of the business were identified. The data were monthly collected from January 2004 to December 2005. The gross margin, net margin, and the result (profit or loss) were taken into consideration as indicators of economic efficiency. The component items of the effective operational cost that had the greatest influence upon the costs of dairy business were, in decreasing order, feeding (55.9\%), labor (19.1\%), different expenses (11.3\%), energy (7.1\%), health (4.9\%), milking $(0.74 \%)$, taxes $(0.74 \%)$, and artificial insemination $(0,23 \%)$. The net margin and the positive result show the farmers have conditions to produce in middle and long terms, with consequent capitalization. Although, the results of the study can not be extrapolated, since they do not represent a random sampling.
\end{abstract}

Keywords: dairy cattle, production cost, breakeven point, profitability analysis

\section{INTRODUÇÃO}

Diversas transformações têm contribuído para que os produtores de leite reflitam sobre a necessidade de administrarem bem a atividade, tornando-a mais eficientes e, conseqüentemente, competitiva, assumindo, assim, posição de

Recebido em 14 de setembro de 2007

Aceito em 9 de janeiro de 2008

E-mail: malopes@ufla.br

Apoio: FAPEMIG (SHA 80894/03) empresário, independente do tamanho da propriedade.

Os dados obtidos da apuração dos custos de produção têm sido utilizados para diferentes finalidades, tais como: estudo da rentabilidade da atividade leiteira; redução dos custos controláveis; planejamento e controle das 
operações da propriedade; identificação e determinação da rentabilidade do produto; identificação do ponto de equilíbrio; e instrumento de apoio ao produtor no processo de tomada de decisões seguras e corretas (Lopes e Carvalho, 2000). Dados de custo de produção têm sido utilizados também para cálculo dos valores econômicos para características de gado de leite (Madalena, 2000; Martins et al., 2003; Bueno et al., 2004), bem como para quantificar ineficiências econômicas (Bravo-Ureta e Rieger, 1991; Tupy e Yamaguchi, 2002). De acordo com Tupy et al. (2003), uma vez quantificada a ineficiência, seus fatores determinantes poderão ser identificados, e muitos prejuízos evitados.

O objetivo desta pesquisa foi estudar a rentabilidade da atividade leiteira na região de Lavras/MG nos anos 2004 e 2005 e, ainda, identificar os componentes que exerceram maior influência sobre os custos finais da atividade.

\section{MATERIAL E MÉTODOS}

Os dados utilizados foram coletados entre janeiro de 2004 e dezembro de 2005, em 17 propriedades leiteiras, selecionadas por amostragem não-probabilística por julgamento (Mattar, 2005). Utilizou-se esse sistema de amostragem para compor uma amostra que representasse a diversidade de propriedades leiteiras localizadas na região de Lavras, sul do estado de Minas Gerais.

A pesquisa considerou duas etapas diferentes no levantamento das informações. Em uma etapa, por meio de um questionário e com uma caderneta de campo, foi realizado o inventário completo dos bens das propriedades estudadas, apurando valor e vida útil de cada ativo, sendo, posteriormente, alocados em um dos seguintes grupos: benfeitorias, máquinas, veículos, equipamentos e implementos, ferramentas e rebanho. Nas situações nas quais o pecuarista não dispunha de informações referentes ao valor e à data de aquisição, para a estimativa dos valores atuais, bem como da vida útil restante, foi adotado o critério proposto por Lopes et al. (2004a).

Quanto às benfeitorias, cada uma foi medida, sendo atribuído um estado de conservação e registrado um resumo do memorial descritivo, objetivando auxiliar na estimativa do valor atual. Em função da área, do estado de conservação e do padrão de acabamento, foi estimado um valor por $\mathrm{m}^{2}$ de construção. $\mathrm{O}$ valor atual utilizado foi produto do valor do $\mathrm{m}^{2}$ pela área da benfeitoria (Lopes et al., 2004a).

$\mathrm{Na}$ outra etapa, as propriedades foram visitadas no início de cada mês para coleta de dados referentes às produções, despesas realizadas $\mathrm{e}$ receitas apuradas no mês anterior. A coleta de dados deu-se em cadernetas de campo especificamente preparadas para esse fim. Tais dados foram cadastrados no software Custo Bovino Leite $^{\circledR}$ (Lopes et al., 2002a), aplicativo utilizado para o processamento eletrônico dos dados bem como para análise de rentabilidade dos sistemas de produção. Tal software contemplou as duas estruturas de custo de produção: custo total de produção, que envolve o custo fixo e variável, e custo operacional, proposta por Matsunaga et al. (1976).

Os itens que compõem o custo operacional efetivo de produção do leite foram divididos em grupos: mão-de-obra, alimentação, sanidade, reprodução, ordenha, impostos, energia e despesas diversas (Lopes et al., 2004a; Lopes et al., 2006).

Os índices produtivos e econômicos foram comparados por meio de análises descritivas, utilizando o aplicativo MS Excel ${ }^{\circledR}$, e agrupados em tabelas, objetivando uma melhor comparação, discussão e apresentação dos resultados (Lopes et al., 2004b).

\section{RESULTADOS E DISCUSSÃO}

Um resumo dos recursos disponíveis nas 17 propriedades leiteiras da região é apresentado na Tab. 1. Tais recursos foram úteis nas análises e discussões dos resultados encontrados nesta pesquisa. $\mathrm{Na}$ Tab. 2 pode ser observado um resumo da análise de rentabilidade da atividade leiteira dessas propriedades. Pelos altos valores de desvio-padrão, pode-se constatar que as propriedades estudadas foram bem diferentes entre si. 
Tabela 1. Recursos disponíveis em 17 propriedades leiteiras da região de Lavras, MG, entre janeiro de 2004 e dezembro de 2005

\begin{tabular}{lcc}
\hline Especificação & Média & Desvio-padrão \\
\hline Valor do patrimônio sem terra (R\$) & $81.244,14$ & $43.951,14$ \\
Valor do patrimônio em terra (R\$) & $212.593,41$ & $159.478,09$ \\
Área (ha) & 77,76 & 53,03 \\
Número de animais em lactação/dia (matrizes) & 38,13 & 21,70 \\
Mão-de-obra (quantidade de serviços/dia) & 2,62 & 2,07 \\
\hline
\end{tabular}

Tabela 2. Resumo da análise de rentabilidade da atividade leiteira de 17 propriedades da região de Lavras, MG, em R\$, entre janeiro de 2004 e dezembro de 2005

\begin{tabular}{lcc}
\hline Especificação & Média & Desvio-padrão \\
\hline Receita & $196.692,22$ & $189.348,84$ \\
Leite & $169.706,75$ & $171.497,53$ \\
Animais & $20.686,38$ & $23.507,58$ \\
Subprodutos & $6.299,09$ & $17.723,10$ \\
Custo operacional total (COT) & $148.838,60$ & $144.516,95$ \\
Custo operacional efetivo (COE) & $132.884,25$ & $141.128,84$ \\
Custo com depreciação & $12.523,76$ & $7.661,11$ \\
Mão-de-obra familiar & 3430,59 & $4.045,23$ \\
Custo total (CT) & $190.276,16$ & $170.456,82$ \\
Custo fixo (CF) & $51.699,41$ & $34.020,87$ \\
Remuneração da terra & $29.696,86$ & $22.541,88$ \\
Remuneração do capital investido & $8.975,10$ & $5.435,41$ \\
Remuneração do empresário & - & - \\
Impostos & 503,68 & 367,47 \\
Depreciação & $12.523,76$ & $7.661,11$ \\
Custo variável (CV) & $138.576,75$ & $141.533,95$ \\
Custo operacional efetivo (s/impostos) & $132.380,57$ & $141.059,10$ \\
Mão-de-obra familiar & $3.430,59$ & $4.045,23$ \\
Remuneração do capital de giro & $2.765,59$ & $2.375,04$ \\
Margem bruta & $63.807,96$ & $63.440,37$ \\
Margem líquida & $47.853,61$ & $61.318,49$ \\
Resultado (lucro ou prejuízo) & $6.416,06$ & $52.824,18$ \\
Margem bruta /kg leite & 0,22 & 0,13 \\
Margem líquida /kg leite & 0,14 & 0,15 \\
Resultado (lucro ou prejuízo) /kg leite & $(0,002)$ & 0,16 \\
Lucratividade (\%) & $3,26 \%$ & 25,67 \\
Rentabilidade (\%) & $1,80 \%$ & 7,46 \\
Quantidade de leite (kg) & $322.001,41$ & $295.440,73$ \\
\hline
\end{tabular}

A receita total média durante o período de estudo foi de $\mathrm{R} \$ 196.692,22 \pm \mathrm{R} \$ 189.348,84$, o que correspondeu à soma dos valores apurados com a venda de leite $(85,9 \%)$, de animais $(12,2 \%)$ e de subprodutos (1,9\%) (Tab. 3). Desse modo, $85,9 \%$ e $12,2 \%$ da contribuição da receita para venda de leite e animais, respectivamente, estão dentro da média encontrada por outros pesquisadores. Quanto à venda de subprodutos (esterco), o valor obtido de $1,9 \%$ está muito abaixo devido ao fato de apenas cinco das 17 propriedades terem comercializado o esterco. A maioria utilizou-o como adubo orgânico em capineiras. De acordo com Lopes et al. (2004a), o fato de o esterco ser utilizado nas próprias propriedades, embora em um primeiro momento signifique redução da receita, representou também redução nas despesas com manutenção das capineiras. Nas visitas às propriedades, foi observado que há bastante desperdício desse subproduto, devido às condições inadequadas de armazenamento. Tal fato pode também justificar 
a pequena comercialização e, conseqüentemente, a diminuição da rentabilidade.

A produtividade de leite por ha/ano, de $2.070,36 \mathrm{~kg}$, é um valor muito pequeno quando comparado aos encontrados por Gomes (1997), Schiffler et al. (1999) e Lopes et al. (2001), porém pouco abaixo do obtido por Lopes et al. (2004a). Esse índice aliado ao da quantidade de matrizes por ha $(0,75)$ evidenciam que as áreas estão com suas capacidades de utilização ociosas. A produção média diária de leite foi de $441,10 \mathrm{~kg}$, com produtividade de $10,36 \mathrm{~kg}$ por matriz em lactação.

Em três propriedades o custo variável médio foi mais elevado que o preço de venda do leite, não se permitindo calcular o ponto de equilíbrio, ao considerar que a produção de 14 das 17 propriedades, foi de $721,60 \mathrm{~kg}$. Nestes, a produção média diária foi de $258,44 \mathrm{~kg}$. Esse índice evidencia que esforços gerenciais e até mesmo tecnológicos devam ser feitos objetivando aumentar a média diária sem aumentar o custo variável médio, pois, se este for majorado, aumentará ainda mais o ponto de equilíbrio. De acordo com Lopes et al. (2004a), uma alternativa é aumentar a eficiência produtiva, ou seja, a produtividade por matriz, otimizando, assim, as despesas com mão-deobra, medicamentos, inseminação artificial, impostos fixos, energia e diversas.
O custo operacional total (COT) de $\mathrm{R} \$ 148.838,60$ foi obtido pela soma do custo operacional efetivo, $\mathrm{R} \$ 132.884,25$, que foi $\mathrm{o}$ desembolso, com o custo de depreciação dos bens patrimoniais, $\mathrm{R} \$ 12.523,76$, e com a remuneração da mão-de-obra familiar, $\mathrm{R} \$ 3.430,59$ (Tab. 2). Embora não seja um desembolso, o valor referente à depreciação representa uma reserva de caixa que deveria ser feita para se repor os bens patrimoniais ao final de sua vida útil. A receita do período permitiu que essa reserva fosse feita e que a mão-de-obra familiar fosse remunerada. Isso significa que ao final da vida útil do bem, permanecendo constantes as condições atuais, o pecuarista teria recursos monetários para a aquisição de um novo bem substituto, não havendo uma descapitalização a médio prazo.

A depreciação foi responsável por $11,0 \%$ do COT, mais baixo que os $14,7 \%$ obtidos por Lopes et al. (2004a) e aos $15,0 \%$ obtidos por Lopes et al. (2006). Embora na literatura não exista informação sobre qual seria um bom valor para esse indicador técnico, pode-se dizer que a eficiência de utilização dos bens do patrimônio dos sistemas de produção estudados nesta pesquisa foi superior ao sistema estudado pelos pesquisadores mencionados.

Tabela 3. Contribuição de cada item na receita em 17 propriedades da região de Lavras, MG, em \%, entre janeiro de 2004 e dezembro de 2005 e contribuição de outros estudos

\begin{tabular}{lcccccc}
\hline \multirow{2}{*}{ Especificação } & \multicolumn{3}{c}{ Atual estudo (\%) } & \multicolumn{3}{c}{ Outros estudos (\%) } \\
\cline { 2 - 7 } & Média & DP & 1 & 2 & 3 & 4 \\
\hline Venda de leite & 85,90 & 15,2 & 80,9 & 95,8 & 82,5 & 86,9 \\
Venda de animais & 12,15 & 13,6 & 16,0 & 4,1 & 17,4 & 12,4 \\
Venda de subprodutos & 1,95 & 3,7 & $*$ & $*$ & $*$ & 0,6 \\
Equipamentos obsoletos & 0,0 & 0,0 & 2,4 & $*$ & $*$ & 0,0 \\
\hline
\end{tabular}

$\mathrm{DP}=$ desvio-padrão. 1) Lopes et al. (2001); 2) Lopes et al. (2002b); 3) Almeida Júnior et al. (2002); 4) Lopes et al. (2004a);* não mencionado

O custo operacional efetivo de $\mathrm{R} \$ 132.884,25 \pm \mathrm{R} \$ 141.128,84$ representou o desembolso médio no período de 24 meses para custear a atividade. Os itens que compõem o custo operacional efetivo de produção do leite foram divididos em grupos, cada qual responsável pelos percentuais encontrados na Tab. 4. A divisão das despesas em grupos, de acordo com Lopes e Lopes (1999), permite o monitoramento das despesas da propriedade, auxiliando o técnico e o produtor em uma análise mais detalhada. 
Tabela 4. Contribuição de cada item no custo operacional efetivo de 17 propriedades leiteiras da região de Lavras, MG, em \%, entre janeiro de 2004 e dezembro de 2005 e contribuição de outros estudos

\begin{tabular}{lccccccc}
\hline & \multicolumn{3}{c}{ Atual estudo } & \multicolumn{5}{c}{ Outros estudos (\%) } \\
Especificação & Média (\%) & DP & 1 & 2 & 3 & 4 & 5 \\
\hline Mão-de-obra & 19,14 & 10,41 & 15 & 25,81 & 12,33 & 18,95 & 13,37 \\
Alimentação & 55,86 & 11,16 & 55 & 41,74 & 58,35 & 65,33 & 59,95 \\
Sanidade & 4,90 & 2,31 & 6 & 6,29 & 5,62 & 2,88 & 3,76 \\
Inseminação artificial & 0,23 & 0,32 & 5 & 4,78 & 1,35 & 1,02 & 0,22 \\
Ordenha & 0,74 & 0,99 & 1 & 3,07 & 1,04 & 1,87 & 0,88 \\
Impostos (ITR e IPVA) & 0,74 & 0,73 & $*$ & 0,17 & 0,75 & $*$ & 0,83 \\
Energia & 7,10 & 4,06 & $*$ & $*$ & $*$ & $*$ & 10,16 \\
Despesas diversas & 11,29 & 4,64 & 18 & 18,14 & 20,64 & 9,96 & 10,87 \\
\hline
\end{tabular}

DP= desvio-padrão. 1) Berg e Katsman (1998); 2) Lopes et al. (2001); 3) Almeida Júnior et al. (2002); 4) Lopes et al. (2002b); 5) Lopes et al. (2004a); *não mencionado

Quanto à mão-de-obra, os 19,1\% estão acima da média encontrada por outros pesquisadores (Tab. 4), evidenciando que parte dela está ociosa. Tal evidência é corroborada pela baixa relação vaca:homem de 16,28:1 encontrada neste estudo, pois, se a relação é baixa, a contratação aumenta, $\mathrm{e}$, conseqüentemente, o percentual de despesas referentes à mão-de-obra tende a aumentar. Lopes et al. (2001), ao realizarem a análise de rentabilidade de uma propriedade leiteira com resultados altamente insatisfatórios, encontraram uma relação vaca:homem de 7:1, sendo a mãode-obra responsável por $25,8 \%$ do total das despesas operacionais efetivas. Jarret (1997) preconizou uma relação vaca:homem de $66: 1$ e 111:1, para sistemas de produção com 300 e 1000 vacas, respectivamente. A produção diária de leite por mão-de-obra permanente de $167,75 \mathrm{~kg}$ está muito aquém das obtidas por Schiffer et al. (1999) $(246,12 \mathrm{~kg})$ e além dos $80 \mathrm{~kg}$ encontrados por Lopes et al. (2001).

A alimentação foi responsável por $55,9 \%$ das despesas operacionais efetivas. Esse percentual está dentro da média encontrada por outros pesquisadores. Vale salientar que todas as propriedades utilizaram suplementação concentrada durante todo o ano e, na estação seca, suplementação volumosa e concentrada. Em três das 17 propriedades, as vacas foram totalmente confinadas.

As despesas com medicamentos representaram $4,9 \%$ do custo operacional efetivo, valor abaixo da média encontrada por outros pesquisadores. Quanto às despesas com inseminação artificial sêmen, nitrogênio líquido, além de outros materiais -, o percentual obtido de 0,23 está muito abaixo da média encontrada por outros pesquisadores, devido ao fato de apenas sete das 17 propriedades leiteiras terem adotado essa tecnologia. A média de tais despesas nas sete propriedades foi de $0,55 \%$

As despesas com produtos utilizados na ordenha representaram $0,74 \%$ das despesas operacionais efetivas, valor abaixo de todos os encontrados na literatura. A explicação para tal ocorrência é que quatro das 17 propriedades não tiveram nenhum gasto com esses materiais, mostrando pouca preocupação com a obtenção higiênica do leite. Impostos considerados fixos como o ITR e o IPVA representaram apenas $0,74 \%$, evidenciando que o não pagamento desses em nada ajudaria o pecuarista.

No grupo energia, que representou $7,1 \%$, foram consideradas as despesas com energia elétrica e combustíveis. Foram consideradas despesas diversas aquelas que não se enquadram nos grupos mencionados anteriormente, tais como frete do leite, taxas e impostos variáveis em função da produção, despesas com manutenção de benfeitorias, máquinas e equipamentos. O valor de $11,3 \%$ está abaixo dos mencionados por Lopes et al. (2001) e Almeida Júnior et al. (2002) porque esses pesquisadores incluíram as despesas com energia elétrica e combustíveis no grupo de despesas diversas.

O custo total foi de $\mathrm{R} \$ 190.276,16$. A diferença entre o custo total e a receita, de $\mathrm{R} \$ 6.416,06$ (R\$196.692,22- R\$190.276,16), mostra que todos os custos variáveis puderam ser pagos, que a reserva referente à depreciação pôde ser 
realizada, e que o capital investido em bens e terra foi completamente remunerado.

Os custos fixos, que totalizaram $\mathrm{R} \$ 51.699,41 \mathrm{e}$ representaram $27,2 \%$ do custo total, são altos quando comparados aos 16,1\% encontrados por Almeida Júnior et al. (2002). Estes resultados evidenciam que os investimentos certamente se encontram dimensionados para uma produção de leite muito maior que a média encontrada. Segundo Lopes et al. (2001), independentemente da quantidade produzida, não havendo aquisição nem venda de bens e nem aumento de impostos, os custos fixos permanecerão constantes. Para que os mesmos sejam menos representativos no custo total, tanto a produção como a produtividade devem ser aumentadas, atingindo uma economia de escala. Os custos variáveis totalizaram $\mathrm{R} \$ 138.576,75$, representando $72,8 \%$ do custo total.

Observa-se pelos indicadores de eficiência econômica margem bruta, margem líquida e resultado - receita bruta menos custo total -, que tais resultados foram satisfatórios (positivos), evidenciando que a atividade leiteira tem condições de sobreviver no curto, médio e longo prazo (Tab. 2), com conseqüente capitalização dos pecuaristas.

Nenhuma das 17 propriedades estudadas teve margem bruta negativa, evidenciando que o que foi apurado com a venda de leite, animais e subprodutos (esterco) foi suficiente para cobrir as despesas operacionais. A margem líquida foi negativa em duas propriedades leiteiras. Quando analisado pelo indicador de eficiência econômica, este resultado foi positivo em apenas sete das 17 propriedades estudadas, evidenciando que nessas a atividade conseguiu remunerar o capital. A lucratividade foi de $3,3 \%$, e a rentabilidade de $1,8 \%$.

$\mathrm{Na}$ Tab. 5 apresentam-se os custos médios de produção por quilograma de leite. $\mathrm{O}$ valor médio de venda do leite, $\mathrm{R} \$ 0,51$, foi suficiente para cobrir as despesas operacionais efetivas, $\mathrm{R} \$ 0,39$, as operacionais totais, $\mathrm{R} \$ 0,46$ e as variáveis, $\mathrm{R} \$ 0,42$. Quando foi tomado como referência o custo total - soma do custo fixo e variável -, de $\mathrm{R} \$ 0,60$, a diferença de $\mathrm{R} \$ 0,09(\mathrm{R} \$ 0,51-\mathrm{R} \$ 0,42)$ foi suficiente para cobrir apenas $47,4 \%$ das despesas fixas, nas quais está incluída a remuneração do capital investido.

$\mathrm{Na}$ Tab. 6 apresentam-se os custos médios de produção por quilograma de "leite virtual". O termo "leite virtual", criado por Lopes e Lopes (1999), significa a quantidade de leite, em kg, resultante da conversão dos valores apurados com as vendas de animais. Esse valor pode ser tomado como referência para o produtor avaliar se a atividade leiteira, principalmente a cria e recria de animais, está sendo viável economicamente. Neste caso, o valor médio de venda de $\mathrm{R} \$ 0,51$ foi suficiente para cobrir as despesas operacionais totais, $R \$ 0,37$, bem como as despesas referentes ao custo total, $\mathrm{R} \$ 0,50$.

Tabela 5. Custo médio de produção, por quilograma de leite, de 17 propriedades leiteiras da região de Lavras, MG, em R\$, entre janeiro de 2004 e dezembro de 2005

\begin{tabular}{lcc}
\multicolumn{1}{c}{ Especificação } & Média & Desvio-padrão \\
\hline Custo operacional total & 0,46 & 0,13 \\
Custo operacional efetivo & 0,39 & 0,12 \\
Custo total & 0,60 & 0,13 \\
Custo fixo & 0,19 & 0,08 \\
Custo variável & 0,42 & 0,13 \\
Preço médio de venda do leite & 0,51 & 0,03 \\
\hline
\end{tabular}

Dentro do preconizado por Lopes (2003), considerando um custo total do leite de $\mathrm{R} \$ 0,60$ (Tab. 5) e o valor de venda de $\mathrm{R} \$ 0,51$, a atividade leiteira não foi viável economicamente, pois o produtor teve prejuízo de $\mathrm{R} \$ 0,09 / \mathrm{kg}$ de leite $(\mathrm{R} \$ 0,51-\mathrm{R} \$ 0,60)$. Nesse caso específico, produzir leite não foi uma atividade recomendável. Nesse custo total de $\mathrm{R} \$ 0,60 / \mathrm{kg}$ de leite, foram computadas todas as despesas, inclusive a da criação de bezerras e de outras categorias de animais. Nesse período, os produtores pesquisados venderam alguns animais. O software Cu\$To Bovino Leite, utilizado no processamento eletrônico dos dados 
desta pesquisa, realizou a conversão dos valores apurados com as vendas de animais em leite e calculou o custo total do "leite virtual" que, nesse caso, foi de $\mathrm{R} \$ 0,50$ (Tab. 6). Assim, quando se considerou a venda de animais, a atividade foi rentável em $\mathrm{R} \$ 0,01 / \mathrm{kg}$ de leite $(\mathrm{R} \$ 0,51$ - $\mathrm{R} \$ 0,50)$. Este resultado mostra que, em algumas situações, pode ser verdadeira a afirmação comum feita por muitos produtores de leite: "produzir leite é mau negócio; o que é bom negócio são as crias; as crias, sim, valem a pena". Os resultados encontrados nesta pesquisa que apontam uma margem bruta, líquida e um resultado positivo (Tab. 2), mesmo sendo o custo total inferior ao preço de venda (Tab. 5), vêm confirmar a importância e aplicabilidade da estimativa do "leite virtual".

Tabela 6. Custo médio de produção por quilograma de "leite virtual", de 17 propriedades leiteiras da região de Lavras, MG, em R\$, entre janeiro de 2004 e dezembro de 2005

\begin{tabular}{lcc}
\hline Especificação & Média & Desvio-padrão \\
\hline Custo operacional total & 0,37 & 0,09 \\
Custo operacional efetivo & 0,33 & 0,10 \\
Custo total & 0,50 & 0,10 \\
Custo fixo & 0,16 & 0,07 \\
Custo variável & 0,34 & 0,10 \\
Preço médio de venda do leite & 0,51 & 0,03 \\
\hline
\end{tabular}

\section{CONCLUSÕES}

A margem líquida e o resultado positivo indicam que os pecuaristas têm condições de produzir a médio e a longo prazo, com conseqüente capitalização. Estes resultados não podem ser extrapolados por não se tratar de uma amostra probabilística. Os itens componentes do custo operacional efetivo que exerceram maior influência sobre os custos da atividade leiteira foram, em ordem decrescente, alimentação, mão-de-obra, despesas diversas, energia, sanidade, ordenha, impostos e inseminação artificial.

\section{REFERÊNCIAS BIBLIOGRÁFICAS}

ALMEIDA JUNIOR, G.A.; LOPES, M.A.; PINATTO, F. Efeito da venda de animais na rentabilidade de um sistema intensivo de produção de leite tipo B no estado de São Paulo In: REUNIÃO ANUAL DA SOCIEDADE BRASILEIRA DE ZOOTECNIA, 39., 2002, Recife. Anais... Recife: SBZ, 2002. (CD, 4p.).

BERG, H.V.D.; KATSMAN, T. Custos comparando despesas na produção do leite. Bol. Leite, v.5, p.3, 1998.

BRAVO-URETA, B.E.; RIEGER, L. Dairy farm efficieny measurement using stochastic frontiers and neoclassical duality. Am. Agric. Econ., v.73, p.421-426, 1991.
BUENO, P.R.B.; RORATO, P.R.N.; DÜRR, J.W. et al. Valor econômico para componentes do leite no estado do Rio Grande do Sul. Rev. Bras. Zootec., v.33, Supl. 3, p.2256-2265, 2004.

GOMES, S.T. Indicadores de eficiência técnica e econômica na produção de leite. São Paulo: FAESP, 1997, 178p.

JARRET, J. Produção de leite de qualidade. In: SIMPÓSIO INTERNACIONAL SOBRE PRODUÇÃO INTENSIVA DE LEITE, 3., 1997, Belo Horizonte. Anais... Belo Horizonte: FMVZUSP, 1997. p.34-45.

LOPES, M.A. Sistemas computacionais para cálculo do custo de produção do leite e carne. Lavras: FAEPE/PROEX. 2003. 35p.

LOPES, M.A.; ALMEIDA JUNIOR, G.A.; CARVALHO, F.C. et al. Estudo da rentabilidade de uma propriedade de leite tipo B no estado de São Paulo. In: CONGRESSO BRASILEIRO DE ADMINISTRAÇÃO RURAL, 4., 2001. Goiânia. Anais... Goiânia: ABAR, 2001. 8p. (CD).

LOPES, M.A.; CAMPELLO, R.P.; CARVALHO, F.M. et al. Custo Bovino Leite 1.0: software de controle de custos para a atividade leiteira. Rev. Bras. Agroinf. v.4, p.102115, 2002a.

LOPES, M.A.; CARMO, E.A.; LIMA, A.L.R. et al. Análise de rentabilidade de uma empresa com 
opção de comercialização de queijo ou leite. Arq. Bras. Med. Vet. Zootec., v.58, p.642-647, 2006.

LOPES, M.A.; CARVALHO, F.M. Custo de produção do leite. Lavras: UFLA, 2000. 42p. (Boletim Agropecuário, 32).

LOPES, M.A.; LIMA, A.L.; REIS, R.P. Custos de produção de leite: um estudo de caso no sul de MG. In: CONGRESSO BRASILEIRO DE MEDICINA VETERINÁRIA, 24., $2002 b$. Anais... Gramado: SOVERGS, 1 p. (CD).

LOPES, M.A.; LIMA, A.L.R.; CARVALHO, F.M. et al. Controle gerencial e estudo da rentabilidade de sistemas de produção de leite na região de Lavras (MG) Rev. Cienc. Agrotec., v.28, p.883-892. 2004a

LOPES, M.A.; LIMA, A.L.R.; CARVALHO, F.M. et al. Efeito do tipo de sistema de criação nos resultados econômicos de sistemas de produção de leite na região de Lavras (MG). Rev. Cienc. Agrotec., v.28, p.1177-1189, 2004b.

LOPES, M.A.; LOPES, D.C.F. Desenvolvimento de um sistema computacional para cálculo do custo de produção do leite. Rev. Bras. Agroinf. v.2, p.1-12, 1999.

MADALENA, F.E. Valores econômicos para a seleção de gordura e proteína do leite. Rev. Bras. Zootec., v.29, p.678-684, 2000.
MARTINS, G.A.; MADALENA, F.H.; BRUSCHI, J.H. et al. Objetivos econômicos de seleção de bovinos de leite para fazenda demonstrativa na Zona da Mata de Minas Geris. Rev. Bras. Zootec., v.32, p.304-314, 2003.

MATTAR, F.N. Pesquisa de marketing. 6.ed. São Paulo: Atlas, 2005. 352p.

MATSUNAGA, M.; BEMELMANS, P.F.; TOLEDO, P.E.N. et al. Metodologia de custo de produção utilizado pelo IEA. Agric. São Paulo, v.23, p.123-139, 1976.

SCHIFFLER, E.A.; MÂNCIO, A.B.; GOMES, S.T. et al. Efeito da escala de produção nos resultados de produção de leite B no estado de São Paulo. Rev. Bras. Zootec., v.28, p.425-431, 1999.

TUPY, O.; FREITAS, A.R.; ESTEVES, S.N. et al. Eficiência econômica na produção de leite tipo B no estado de São Pulo. Inf. Econ., v.33, 2003.

TUPY, O.; YAMAGUCHI, L.C.T. Identificando benchmarks de leite. Rev. Econ. Sociol. Rural, v.40, p.81-96, 2002. 